\title{
Exact solution of a one-dimensional fermion model with interchain tunneling
}

\author{
R. Z. Bariev ${ }^{(\mathrm{a})}$, A. Klümper ${ }^{(\mathrm{b})}$, A. Schadschneider ${ }^{(\mathrm{c})}$ and \\ J.Zittartz ${ }^{(\mathrm{b})}$ \\ Institut für Theoretische Physik, Universität zu Köln, Zülpicher Strasse 77, D-50937 \\ Köln, Federal Republic of Germany
}

PACS number(s): 71.28.+d, 74.20.-z, 75.10.Lp

Date: 17 May 2018 
Abstract. A fermion model consisting of two chains with interchain tunneling is formulated and solved exactly by the Bethe ansatz method. The interchain tunneling leads to Cooper pair like bound states and a threshold energy is required to overcome the binding energy. The correlation functions manifest superconducting properties. 
Since several years there has been considerable interest in unusual mechanisms for the formation of Cooper pairs because of the possible relevance for high $T_{c}$ superconductors. A simple model showing a pairing transition was recently proposed in [1] to explain the properties of the high-temperature cuprate superconductors. The underlying mechanism leading to the superconducting transition is an interlayer tunneling phenomenon. Although the model in [1] is two-dimensional, it is quite interesting to investigate its one-dimensional variant, as it has been conjectured [2] that properties of the one- and two-dimensional models have common aspects. Exact results in one dimension are often more easily available than in two dimensions and may provide a testing ground for approaches intended for more complex problems.

In this paper we present the exact solution to a new integrable model consisting of two chains with an interchain tunneling which may be considered as the simplest onedimensional variant of the model in [1]. It will be shown that the attractive interaction leads to bound states of Cooper type pairs and the model has superconducting properties.

The Hamiltonian under consideration is the following

$$
\mathcal{H}=-\sum_{j=1}^{L} \mathcal{P}\left[c_{j 1}^{+} c_{j+1,1}+c_{j 2}^{+} c_{j+1,2}-V c_{j 1}^{+} c_{j+1,1}^{+}\left(c_{j 2} c_{j+1,2}+c_{j-1,2} c_{j 2}\right)+\text { h.c. }\right] \mathcal{P}
$$

where the Fermi operator $c_{j \alpha}^{+}$creates an electron at site $j$ with a sublattice index $\alpha(\alpha=1,2), L$ is the number of lattice sites, and h.c. denotes the Hermitian conjugate. We impose the restriction of no simultaneous occupancies of sites on different sublattices which are nearest neighbours, i.e. $(j, 1) ;(j, 2)$ and $(j, 1) ;(j-1,2)$, and $\mathcal{P}$ is the projector on the subspace of allowed states. The Hamiltonian (1) contains two different terms. The first describes the hopping of individual electrons with transition rate set equal to 1 , and the second describes the hopping of pairs of electrons with rate $V(>0)$. The competition of these terms is the main property of the model under consideration. It should be noted that this is similar to the one-dimensional model proposed by Penson and Kolb [3]. We hope that the study of (1) will also help to understand this nonintegrable model.

The exact solution for the eigenstates and eigenvalues of Hamiltonian (1) can be obtained within the framework of the Bethe ansatz method [4-6]. The structure of the Bethe ansatz equations follows from the solution of the two-particle problem. The 
nonvanishing elements of the $S$-matrix are

$$
\begin{aligned}
& S_{11}^{11}(k)=S_{22}^{22}(k)=i \frac{\sinh \eta}{\sin (k+i \eta)} \mathrm{e}^{-i k}, \\
& S_{11}^{22}(k)=S_{22}^{11}(k)=-\frac{\sin k}{\sin (k+i \eta)} \mathrm{e}^{-i k}, \\
& S_{12}^{21}(k)=S_{21}^{12}(k)=\mathrm{e}^{-i k}, \quad \eta=-\ln V .
\end{aligned}
$$

A necessary and sufficient condition for the applicability of the Bethe ansatz method are the Yang-Baxter equations [4]. Since the scattering matrix (2) satisfies these equations, our model (1) is integrable.

The discrete Bethe ansatz equations are derived following the standard procedure [7] by imposing periodic boundary conditions. Each state of the Hamiltonian is specified by one set of charge rapidities $k_{j}$ representing the momenta of the electrons and one set of additional rapidities $\Lambda_{\alpha}$. All rapidities within a given set have to be different, corresponding to Fermi statistics. These rapidities are determined by the Bethe ansatz equations

$$
\begin{aligned}
& \mathrm{e}^{i k_{j} L}=\prod_{l=1}^{N} \exp \left[\frac{i}{2}\left(k_{j}-k_{l}\right)\right] \prod_{\alpha=1}^{M} \frac{\sin \left[\frac{1}{2}\left(k_{j}-\Lambda_{\alpha}\right)+\frac{c}{2} \eta\right]}{\sin \left[\frac{1}{2}\left(k_{j}-\Lambda_{\alpha}\right)-\frac{c}{2} \eta\right]} \\
& \prod_{j=1}^{N} \frac{\sin \left[\frac{1}{2}\left(\Lambda_{\alpha}-k_{j}\right)+\frac{c}{2} \eta\right]}{\sin \left[\frac{1}{2}\left(\Lambda_{\alpha}-k_{j}\right)-\frac{\varsigma}{2} \eta\right]}=-\prod_{\beta=1}^{M} \frac{\sin \left[\frac{1}{2}\left(\Lambda_{\alpha}-\Lambda_{\beta}\right)+\subset \eta\right]}{\sin \left[\frac{1}{2}\left(\Lambda_{\alpha}-\Lambda_{\beta}\right)-\subset \eta\right]} .
\end{aligned}
$$

The energy and the momentum of the corresponding state are given by

$$
E=-2 \sum_{j=1}^{N} \cos k_{j}, \quad P=\sum_{j=1}^{N} k_{j}
$$

We remark that in contrast to [4-6] in our model the electron number on each separate sublattice is not conserved. In the case under consideration the conserved quantities are the total number of electrons $N$ and the number $M$ of pairs of consecutive electrons which are on the same sublattice.

Each eigenstate of the Hamiltonian is specified by sets of rapidities which have to satisfy the Bethe ansatz equations (3). The structure of the solutions of these equations is different for $\eta<0$ and $\eta>0$. In the case $\eta>0$ all $k_{j}$ are real and particles move independently. There are no Cooper pairs in the system. Equations 
(3) are reduced to one set of equations for $k_{j}$

$$
\begin{aligned}
& k_{j} L-\frac{1}{2} \sum_{i=1}^{N}\left(k_{j}-k_{i}\right)+\sum_{i=1}^{N} \Phi\left(k_{j}-k_{i}\right)=2 \pi I_{j}, \\
& \Phi(k)=\frac{k}{2}+\sum_{\nu=1}^{\infty} \frac{\exp (-\nu \eta)}{\nu} \frac{\sin (\nu k)}{\cosh (\nu \eta)} .
\end{aligned}
$$

where $I_{j}$ are integer (half-integer) numbers for odd (even) $N$. (We have included the linear term $k / 2$ in the definition of the function $\Phi(k)$ such that it is identical to the phase function used in [10].) In the thermodynamic limit eqs. $(4,5)$ are replaced by the integral equation for the distribution function of particles $\rho(k)$

$$
2 \pi \rho(k)-\int_{-k_{0}}^{k_{0}} \varphi\left(k-k^{\prime}\right) \rho\left(k^{\prime}\right) d k^{\prime}=1-\frac{1}{2} \rho, \quad \varphi(k)=\Phi^{\prime}(k)
$$

with subsidiary condition for the particle density $\rho$

$$
\int_{-k_{0}}^{k_{0}} \rho(k) d k=\rho=N / L
$$

In the opposite case $\eta<0(V>1)$ pairs of electrons are energetically more favourable as compared to unpaired particles. In the ground state we only have Cooper pair like bound states with finite pairing energy which are characterized by pairs of complex wavenumbers

$$
k_{\alpha}^{ \pm}=\Lambda_{\alpha} \pm \subset|\eta|
$$

Inserting (9) into the Bethe ansatz equations (3) we obtain the following set of equations

$$
\begin{aligned}
& 2 L \Lambda_{\alpha}-2 \sum_{\beta=1}^{M}\left(\Lambda_{\alpha}-\Lambda_{\beta}\right)-\sum_{\beta=1}^{M} \Theta\left(\Lambda_{\alpha}-\Lambda_{\beta} ;|\eta|\right)=2 \pi J_{\alpha}, \\
& E=\sum_{\alpha=1}^{M} e_{0}\left(\Lambda_{\alpha}\right), \quad e_{0}(\Lambda)=-4 \cosh \eta \cos \Lambda, \\
& \Theta(\Lambda ; \eta)=2 \arctan (\operatorname{coth} \eta \tan \Lambda / 2), \quad-\pi<\Theta(\Lambda ; \eta) \leq \pi,
\end{aligned}
$$

where $J_{\alpha}$ are integers or half-odd integers depending on the parity of $M$. In the thermodynamic limit eqs. (10) lead to the integral equation for the distribution function of Cooper pairs $\sigma(\Lambda)$ :

$$
2 \pi \sigma(\Lambda)+\int_{-\Lambda_{0}}^{\Lambda_{0}} \Theta^{\prime}\left(\Lambda-\Lambda^{\prime} ;|\eta|\right) \sigma\left(\Lambda^{\prime}\right) d \Lambda^{\prime}=2\left(1-\frac{1}{2} \rho\right),
$$


with subsidiary condition

$$
\int_{-\Lambda_{0}}^{\Lambda_{0}} \sigma(\Lambda) d \Lambda=\frac{1}{2} \rho .
$$

In order to understand the superconducting properties of the model, we shall investigate the long-distance behaviour of the correlation functions. For this purpose we shall use the methods of two-dimensional conformal field theory $[8,9]$. Our model has just one type of gapless excitations which correspond to "holes" and "particles" in the $I$-distribution or $J$-distribution for the case $\eta>0$ and $\eta<0$, respectively. Therefore, an application of this theory presents no difficulties and can be carried through as in $[10,11]$. The results of these calculations are the following.

For $\eta>0$ the long-distance power-law behaviour of the density-density correlation functions is given by

$$
\langle n(r) n(0)\rangle \simeq \rho^{2}+A r^{-\alpha} \cos (\pi \rho r), \quad n(r)=\sum_{\tau=1,2} c_{r \tau}^{+} c_{r \tau}
$$

The critical exponent $\alpha$ is expressed in terms of the dressed charge $Z$ which in our case is given by

$$
\alpha=Z^{2} / 2, \quad Z=2 \pi \rho\left(k_{0}\right) .
$$

The superconducting properties of the system manifest themselves in the behaviour of the pair correlation functions for which we obtain

$$
G_{p}(r, \tau)=\left\langle c_{r 1}^{+} c_{r+1,1}^{+} c_{0 \tau} c_{1 \tau}\right\rangle \simeq B r^{-\beta}, \quad \beta=2 / Z^{2}=\frac{1}{\alpha} .
$$

In the same way we can consider the case $\eta<0$. The expressions (13)-(15) also hold in this case, but the dressed charge $Z$ is given in terms of the distribution function $\sigma(\Lambda)$

$$
Z=2 \pi \sigma\left(\Lambda_{0}\right)
$$

Comparison of eqs. (13)-(16) with the corresponding results for the model of electrons with correlated hopping [11] permits us to obtain a simple relation between the critical exponents of these models

$$
\begin{aligned}
& \beta(\rho, \eta)=\left(1-\frac{1}{2} \rho\right)^{-2} \beta_{(c)}\left(\frac{1-\rho}{1-\rho / 2}, \eta\right), \quad \eta>0, \\
& \beta(\rho, \eta)=\left(1-\frac{1}{2} \rho\right)^{-2} \beta_{(c)}\left(\frac{\rho}{2-\rho},|\eta|\right), \quad \eta<0, \\
& 0<\rho<1
\end{aligned}
$$


where $\beta_{(c)}$ is the critical exponent of the superconducting correlation function for electrons with correlated hopping [11]. Numerical results for the critical exponent $\beta$ are shown in Fig. 1.

In addition to $(17)$ there is a relation of the charge stiffnesses $D(\rho, \eta)$ and $D_{(c)}(\rho, \eta)$ of both models

$$
\begin{aligned}
& D(\rho, \eta)=\left(1-\frac{1}{2} \rho\right) \mathrm{e}^{-\eta} D_{(c)}\left(\frac{1-\rho}{1-\rho / 2}, \eta\right), \quad \eta>0 \\
& D(\rho, \eta)=\left(1-\frac{1}{2} \rho\right) D_{(c)}\left(\frac{\rho}{2-\rho},|\eta|\right), \quad \eta<0 \\
& 0<\rho<1 .
\end{aligned}
$$

Figs. 2 and 3 show numerical results for the charge stiffness and transport mass of the charge carriers defined by

$$
\frac{m}{m_{e}}=\frac{D^{0}}{D},
$$

where $m_{e}$ is the bare mass and $D^{0}=\frac{2}{\pi} \sin \frac{\pi}{2} \rho$ is the charge stiffness of free fermions. For densities $\rho$ approaching the maximum allowed value 1 the masses diverge due to a (trivial) metal-insulator transition.

The existence of a simple relation between the critical exponents, $\alpha \beta=1$, is certainly a consequence of the universal Luttinger behaviour of critical models with one type of gapless excitations [12]. As our model of interchain tunneling (1) is described by a conformal field theory with central charge $c=1$, the critical exponents depend in a nonuniversal way on the particle density $\rho$ and the interaction strength $\eta$. Although the model of electrons with correlated hopping [11] is different, there exists a mapping of the phase diagram of the first model to the latter one with a simple relation of the phase points and the corresponding values of the exponents $\beta$ and $\beta_{(c)}$, respectively. Note that the mapping is one-to-one, however, the physical properties of the models differ. In contrast to [11] we see that for (1) with couplings $\eta>0$ there are no densities $\rho$ for which $\beta<\alpha$. This means that the dynamics is dominated by the motion of individual electrons rather than by the motion of pairs, and we have no "superconducting" phase for any particle concentration.

For $\eta<0$ the situation is quite different. As in the model with correlated hopping there is a regime for which $\beta<\alpha$, see. Fig. 4 . In this case the pair correlation function has a slower decay than the density-density correlation function and thus dominates. In one-dimensional systems which do not undergo a condensation and do not have 
long-range order such behaviour of correlation functions is the closest analogy to the existence of a superconducting phase [13].

\section{Acknowledgments}

This work has been performed within the research program of the Sonderforschungs-

bereich 341, Köln-Aachen-Jülich. RZB gratefully acknowledges the hospitality of the Institut für Theoretische Physik, Universität zu Köln. 


\section{References}

(a) Permanent address: The Kazan Physico-Technical Institute of the Russian Academy of Sciences, Kazan 420029, Russia

(b) Email: kluemper@thp.uni-koeln.de, zitt@thp.uni-koeln.de

(c) Present address: Institute for Theoretical Physics, State University of New York at Stony Brook, Stony Brook, N Y 11794-3840, U.S.A.

[1] S.Chakravarty, A.Sudbo, P.W.Anderson and S.Strong, Science 261, 337 (1993)

[2] P.W.Anderson, Phys.Rev.Lett. 64, 1839 (1990)

[3] K.Penson and M.Kolb, Phys.Rev.B 33, 1663(1986)

[4] C.N.Yang, Phys.Rev.Lett. 19, 1312 (1967)

[5] E.H.Wu and F.Y.Wu, Phys.Rev.Lett. 20, 1445 (1968)

[6] B.Sutherland, Phys.Rev.B 12, 3795 (1975)

[7] L.A.Takhtajan and L.D.Faddeev, Russ.Math.Surveys 34, 11(1979)

[8] A.A.Belavin, A.M.Polyakov, A.B.Zamolodchikov, Nucl.Phys.B 241, 333 (1984)

[9] J.L.Cardy, Nucl.Phys.B 270 [FS16], 186 (1986)

[10] R.Z.Bariev, A.Klümper, A.Schadschneider and J.Zittartz, J. Phys.A: Math.Gen. 26, 4863 (1993)

[11] R.Z.Bariev, A.Klümper, A.Schadschneider and J.Zittartz, J. Phys.A: Math.Gen. 26, 1249 (1993)

[12] L.P.Kadanoff and A.Brown, Ann. Phys. 121, 318 (1979)

[13] N.M.Bogoliubov and V.E.Korepin, Int.J. Mod.Phys.A 3, 427 (1989) 


\section{Figure captions}

Figure 1. (a) Depiction of the critical exponent $\beta$ in dependence on the particle density $\rho$ for different values of $\eta=0.1,0.5,1,10$. (b) The same as for (a) with negative values of $\eta,|\eta|=0.05,0.1,0.5,1,10$.

Figure 2. Charge stiffness $D_{c}$ and effective mass $m$ of the charge carriers for different interaction strengths $\eta=0.1,0.5,1,2,10$.

Figure 3. Charge stiffness $D_{c}$ and effective mass $m$ for negative values of $\eta,|\eta|=$ $0.1,0.5,1,2$.

Figure 4. Depiction of the phase diagram for $\eta<0$. The solid line separates regions with dominating pair and dominating density-density correlations $(\beta<\alpha$ and $\beta>\alpha)$, respectively. For large $\eta$ the critical density approaches $(4-2 \sqrt{2}) / 3=0.39 \ldots)$. 
$\eta>0$
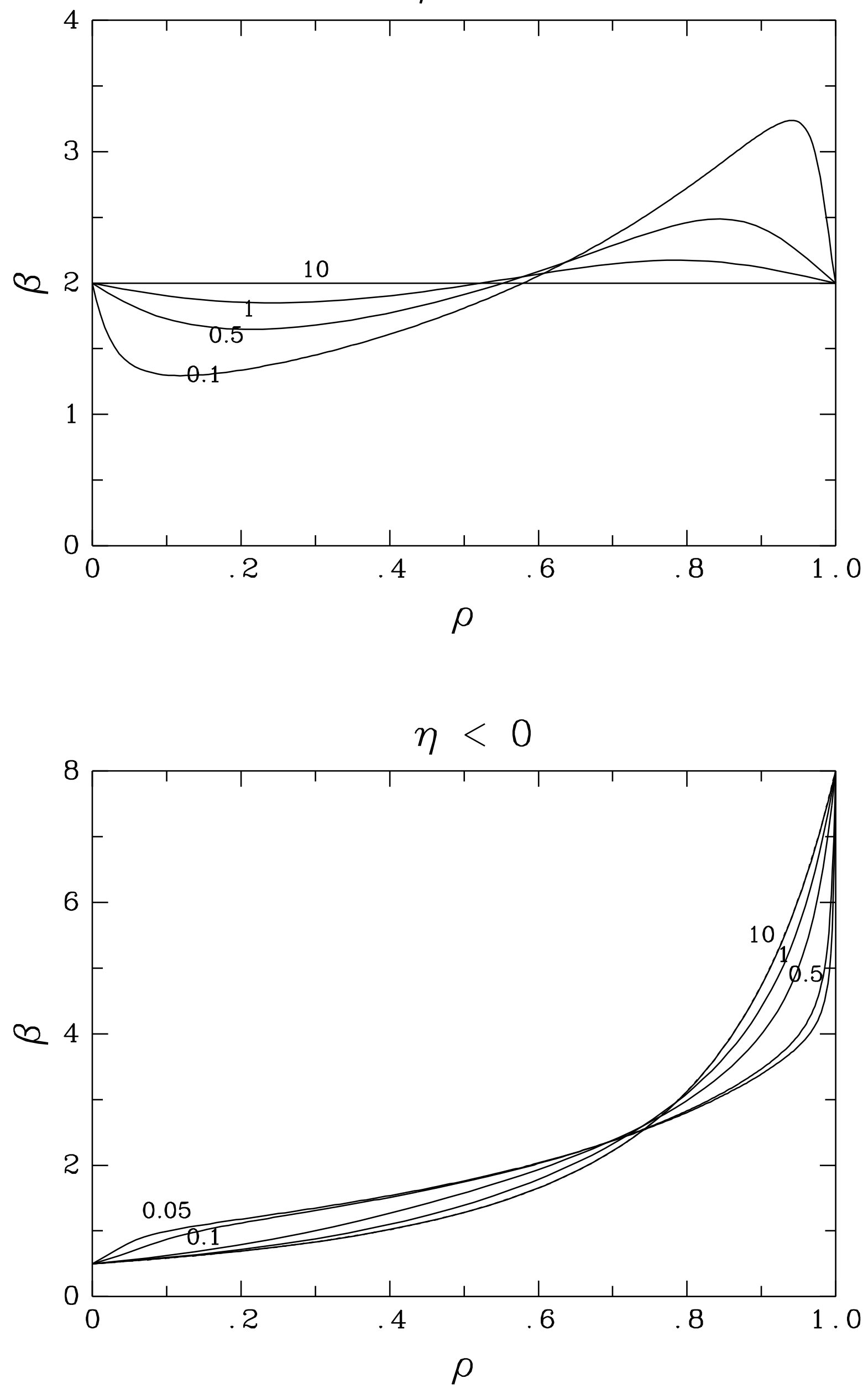

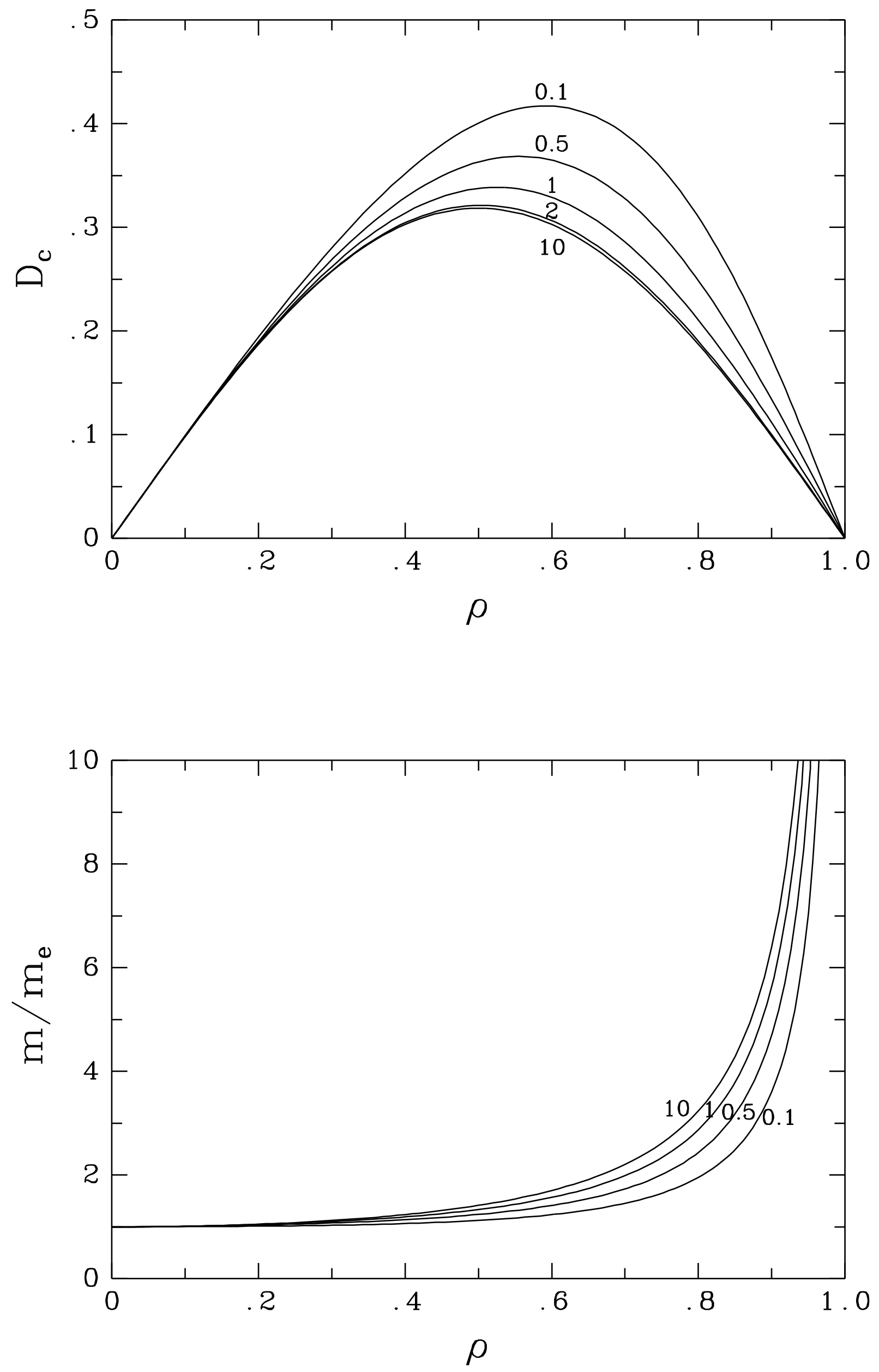

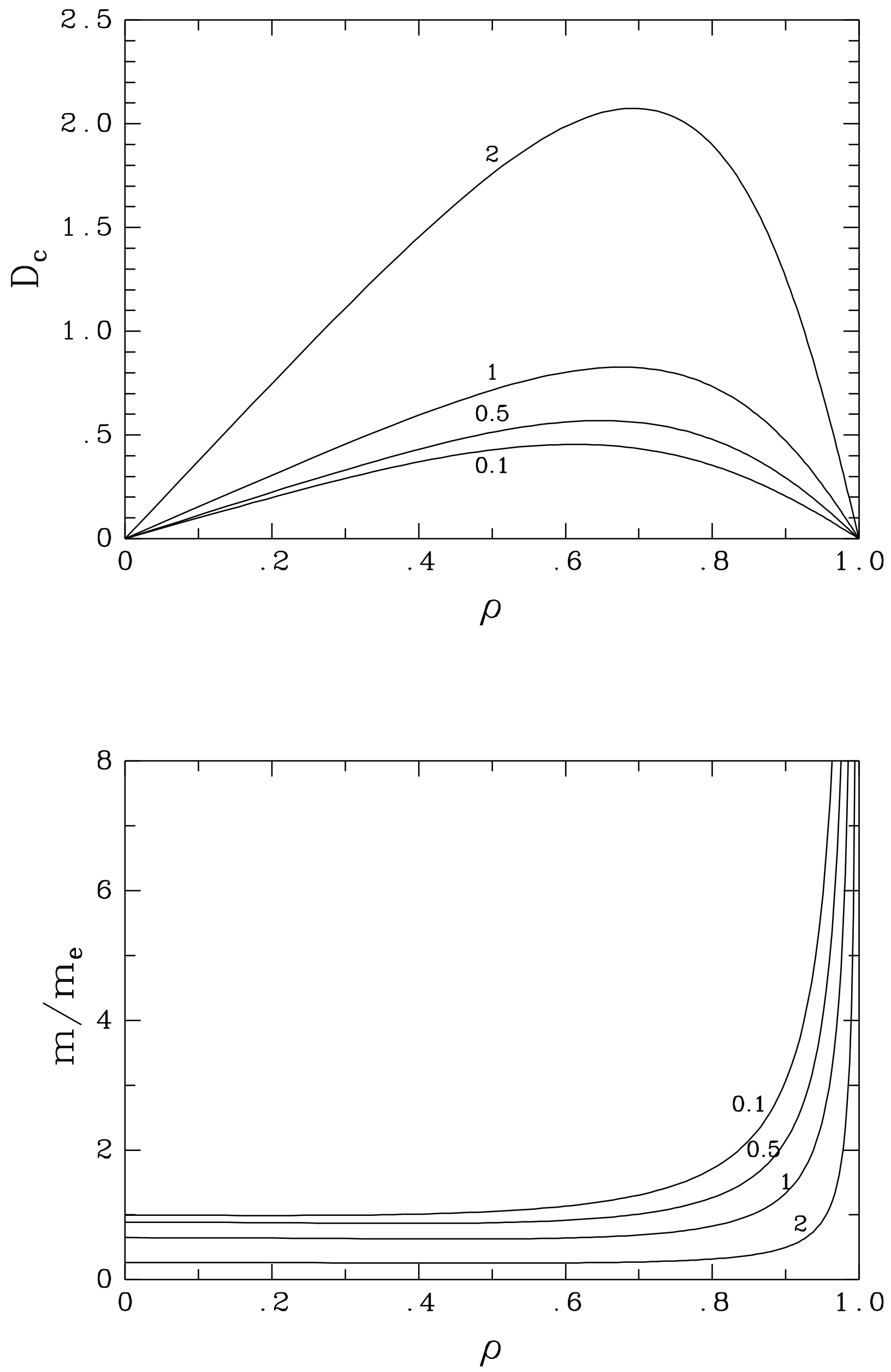


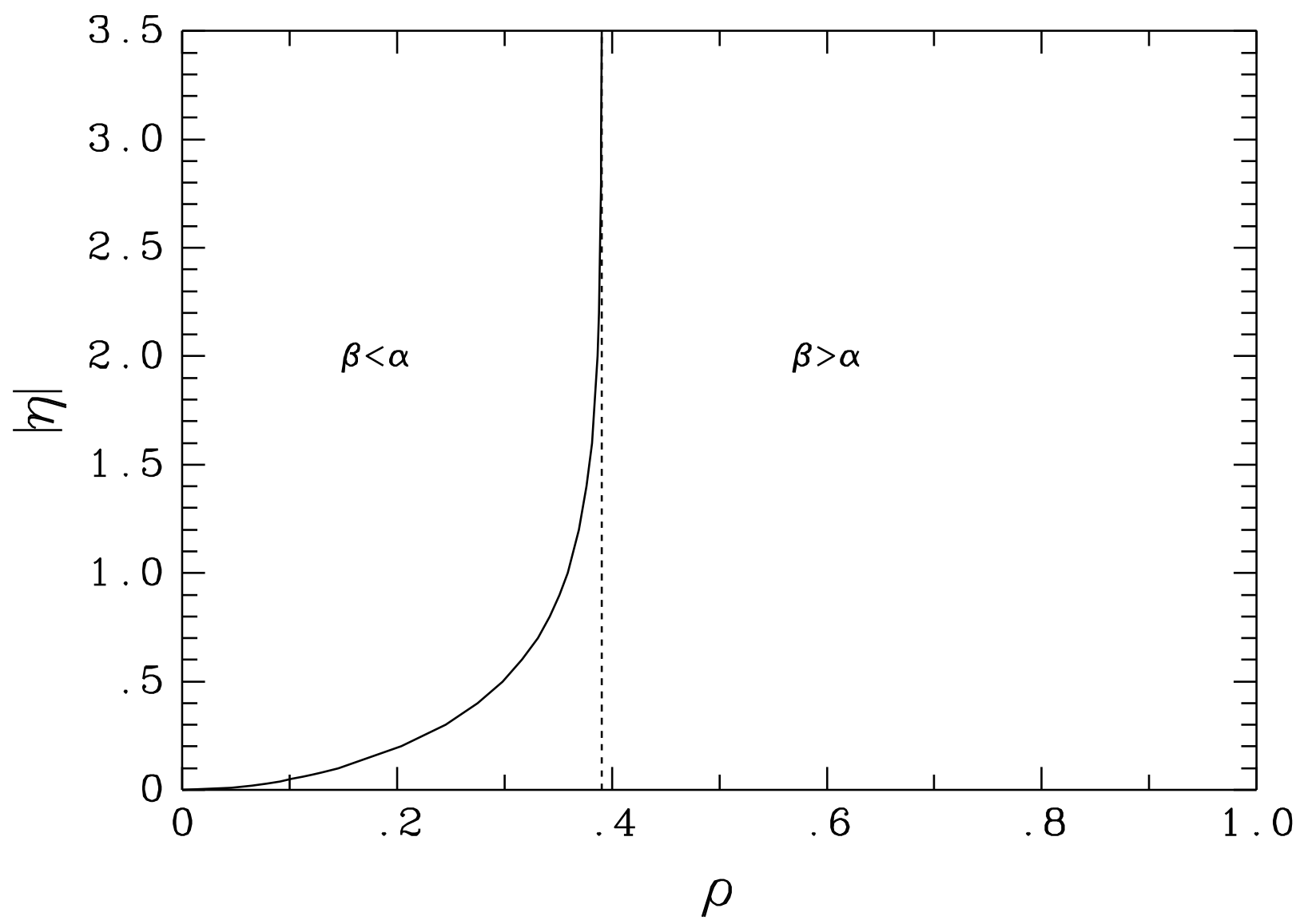

